Journal Club

Editor's Note: These short, critical reviews of recent papers in the Journal, written exclusively by graduate students or postdoctoral fellows, are intended to summarize the important findings of the paper and provide additional insight and commentary. For more information on the format and purpose of the Journal Club, please see http://www.jneurosci.org/misc/ifa_features.shtml.

\title{
Studying Mesodiencephalic Dopaminergic Neuron Development In Vivo to Improve Stem Cell Therapy in Parkinson's Disease
}

\author{
- Jesse V. Veenvliet \\ Max Planck Institute for Molecular Genetics, Department of Developmental Genetics, 14195, Berlin, Germany \\ Review of Fukusumi et al.
}

Parkinson's disease (PD) is characterized by degeneration of mesodiencephalic dopaminergic neurons (mdDAns). Importantly, the disease primarily affects mdDAns of the substantia nigra pars compacta $(\mathrm{SNc})$, leaving mdDAns in the ventral tegmental area (VTA) relatively unaffected (Brichta and Greengard, 2014). A prospective treatment involves replacement of degenerated mdDAns with neurons derived from pluripotent stem cells (PSCs), and many neurodevelopmental studies have focused on the establishment of effective cell replacement strategies (Steinbeck and Studer, 2015). Because the PSC-derived neurons of the SNc subtype (as opposed to those of the VTA subtype) are critical for recovery and proper innervation in $\mathrm{PD}$ models upon grafting (Thompson et al., 2005; Grealish et al., 2010), selective generation of PSCderived SNc-mdDAns is necessary. Efforts to achieve this long-standing goal have been hampered by incomplete understanding of the molecular profiles and programs underlying the generation of SNc- and VTAmdDAns in vivo. Nonetheless, some recent studies have partially unveiled how mdDAn

Received Nov. 24, 2015; revised Jan. 8, 2016; accepted Jan. 11, 2016. J.V.V. is funded by the Alexander von Humboldt Foundation. The author declares no competing financial interests.

Correspondence should be addressed to Jesse V. Veenvliet, Max Planck Institute for Molecular Genetics, Department of Developmental Genetics, Innestrasse 73, 14195, Berlin, Germany. E-mail: veenvlie@molgen.mpg.de.

DOI:10.1523/JNEUROSCI.4285-15.2016

Copyright $\odot 2016$ the authors $\quad 0270-6474 / 16 / 361794-03 \$ 15.00 / 0$ subset-specification is established (Veenvliet and Smidt, 2014; Bodea and Blaess, 2015), but the possibility of using this knowledge to increase the yield of PSCderived SNc-mdDAns has only begun to be exploited. In their recent study, Fukusumi et al. (2015) not only identify a secreted factor, Dickkopf 3 (Dkk3), that specifically promotes the differentiation of SNc-mdDAns in vivo, but also use this information to develop a protocol that increases the fraction of SNc-like mdDAns derived from PSCs.

SNc- and VTA-mdDAns arise from proliferating progenitors in the ventricular zone of the mesodiencephalic floor plate. These progenitors express the morphogenetic factor WNT1 (Brown et al., 2011), which is essential for progenitor proliferation: WNT1 knock-out mice display complete absence or severe depletion of mdDAns (Wurst and Prakash, 2014). The effect of WNT1 on progenitor proliferation is mediated by stimulation of the canonical WNT/ $\beta$-catenin pathway (Joksimovic and Awatramani, 2014). Later in development, mdDAn differentiation and maturation proceeds upon shifting the balance from canonical to noncanonical WNT signaling (Arenas, 2014). WNT signaling is modulated by Dickkopfs. Whereas DKK1/2/4 potently inhibit canonical WNT signaling by binding to LRP5/6, DKK3 does not bind LRP at the cell membrane, and the directionality of its effect on WNT signaling and the un- derlying mechanism are largely unclear (Veeck and Dahl, 2012).

Fukusumi et al. (2015) demonstrated that in the mdDA area, Dkk3 is first detected at embryonic day (E) 11.5. In $D k k 3^{-1-}$ embryos, PITX3, that is normally expressed in developing mdDAns of both the SNc and VTA subtypes, is not induced in a rostrolateral subset of $\mathrm{TH}+$ neurons around E14.5 (TH is the ratelimiting enzyme for dopamine synthesis), when the total number of $\mathrm{TH}+$ mdDAns is still unaffected. Importantly, mdDAns positioned rostrolaterally at E14.5 mainly contribute to the adult $\mathrm{SNc}$, whereas caudomedial-located mdDAns mainly give rise to the VTA (Veenvliet and Smidt, 2014). This programming defect ultimately leads to a decreased number of SNc-mdDAns, but not VTA-mdDAns, from E18.5 onwards. Therefore, the in vivo data suggest that $\mathrm{DKK} 3$ is critical for the induction of PITX3 in developing SNc-mdDAns, and that a failure to do so in the absence of DKK3 ultimately leads to their specific degeneration. These data demonstrate the importance of careful spatiotemporal analysis of the mdDA system in mutant embryos using combinations of multiple mdDA markers in neuro-anatomically defined regions of interest: without the assessment of different subpopulations, the earlier defects in $D k k 3^{-1-}$ embryos would have gone unnoticed, because total $\mathrm{TH}+$ cell numbers were not affected at E14.5. 


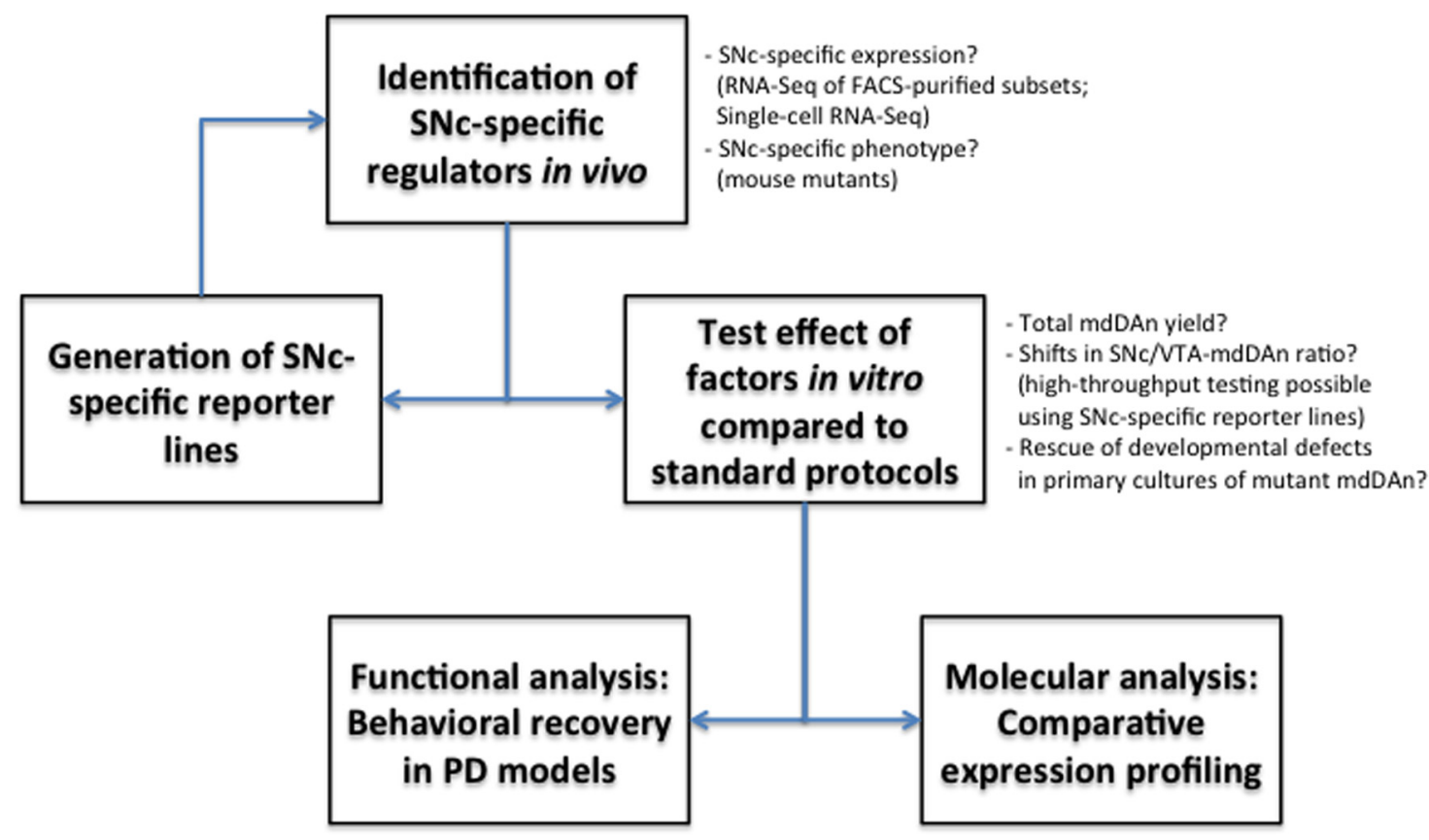

Figure 1. Experimental pipeline for the identification of factors essential for SNc development in vivo and the systematic study of their potential in stem cell therapy for Parkinson's disease. Novel SNc-specific factors can be identified by global expression analysis of mdDAn subsets. Since no specific reporter lines exist to purify developing SNc neurons, these factors can be identified based on transcriptome analysis after anatomical separation, by single-cell RNA-Seq of mdDAns, and/or by analysis of mutants that display SNc-specific degeneration. Novel putative markers can be used to generate reporter lines, which in turn can be used to explore the SNc-specific transcriptome in more detail. In the next phase, putative subset-specific factors, preferably secreted factors for their easy application and therapeutic potential, should be tested for their ability to protect against SNc-mdDAn degeneration in vitro (e.g., in ventral midbrain primary cultures of mutant mice), as well as their ability to increase SNc-mdDAn yield from PSCs in vitro. An appropriate control condition for such an experiment would consist of a standard protocol that generates bona fide mdDAns. It may be critical to test different dosages, combinations, and timing schemes, which will require a high-throughput experimental system. This can be achieved by the use of reporter lines carrying fluorescent proteins under the control of an SNc-specific marker, in combination with a pan-specific mdDAn marker (e.g., PITX3-GFP), which can be used in combination with FACS to assess both mdDAn differentiation efficiency and shifts in SNc/NTA-mdDAn ratio. Finally, behavioral recovery in animal models of PD upon grafting of PSC-derived SNc-mdDAns needs to be demonstrated, and PSC-derived SNc-mdDAns should be compared with their in vivo counterpart in terms of their physiology, epigenome, and transcriptome to assess that they are bona fide SNc-mdDAns.

The specific and exclusive generation of SNc-mdDAns is the holy grail of regenerative medicine, and the identification of compounds that increase SNc-mdDAn yield in vitro would be seminal. It is therefore of utmost interest that Fukusumi et al. (2015) continued to demonstrate that in vitro, treatment with WNT1, DKK3, or both not only increased the percentage of $\mathrm{TH}+$ / PITX3+ PSC-derived mdDAns, but that this was accompanied by an increased percentage of $\mathrm{KCND} 3+/ \mathrm{TH}+$ cells with a concomitant decrease of CALB1+/TH+ mdDAns, indicative of a WNT1/DKK3mediated induction of the rostrolateral SNc fate at the expense of the caudomedial VTA fate in differentiating PSCs (Fukusumi et al., 2015, their Fig. 8). Because PITX3 is widely expressed in all skeletal muscles of the body (L'Honoré et al., 2007), and DKK3 has a decisive function in subtype specification of myogenic cells (Veeck and Dahl, 2012), it is an intriguing possibility that such DKK3mediated subset-specification of cells that display PITX3 expression is a conserved phenomenon.

Surprisingly, the effects of DKK3 loss in vivo were similar to the effect of DKK3 treatment in vitro: whereas in vitro
WNT1/DKK3 treatment subtly increased the percentage of PSC-derived putative SNc-mdDAns at the expense of CALB1+/ $\mathrm{TH}+$ mdDAns (mainly of the VTA subtype), $D k k 3^{-1-}$ mice displayed a marginal decrease of CALB1 +/TH + mdDAns (Fukusumi et al., 2015, their Fig. 8H-N). How can this be explained? A hallmark study demonstrated that strong activation of canonical Wnt signaling [using GSK3 $\beta$ inhibitor CHI99021 (CHIR)] is critical for obtaining bona fide PSC-derived mdDAns (Kriks et al., 2011). It is therefore evident that, in contrast to the wild-type mdDAns taken as a control in vivo, cells in the control condition for the in vitro experiments (omission of WNT1/DKK3) are not bona fide mdDAns. When WNT1 or CHIR treatment is regarded as a control, DKK3 has no additional effect in increasing SNcmdDAn yield (Fukusumi et al., 2015, their Fig. 8G). However, like sole treatment with WNT1, treatment with DKK3 alone shifted the balance to SNc-mdDAns, and may therefore offer another way of increasing SNc-mdDAn yield from PSCs. Whether this is truly an alternative pathway should be elucidated in future studies by unraveling regulatory networks under- lying DKK3 (as compared with WNT1) function in mdDAn development.

Such lack of detailed analysis of pathways conducting the observed effects is a general limitation of the study: although the phenotypical effects of DKK3 loss- or gainof-function are of utmost interest, the exact underlying mechanisms remain unresolved, and it remains unclear whether WNT1 and DKK3 increased mdDAn yield via similar or different mechanisms. For example, WNT1 may act by increasing mdDA progenitor proliferation, whereas DKK3 could affect mdDAn maturation, both eventually resulting in a higher mdDAn yield. Detailed comparative transcriptome analysis of all in vitro treatment conditions could resolve this issue. Fukusumi et al. (2015) demonstrated the power of such analysis by comparative global expression profiling of FACS-purified in vitro and in vivo differentiated PITX3-GFP+ mdDAns, thereby confirming the findings of a recent study that revealed an aberrant transcriptome of induced PSC-derived mdDAns compared with their in vivo counterpart (Roessler et al., 2014). However, whereas comparative transcriptome analysis of all in vitro differentiation conditions would have 
been highly informative, the authors only studied the effects of combinatorial WNT1/ DKK3 treatment as compared with their omission. Although they found some differences, this analysis only provided limited insight into the pathways that conduct the treatment effect (Fukusumi et al., 2015, their Fig. 7 B, C). A likely explanation for this is that analysis was performed on FACSpurified PITX3-GFP+ mdDAns. This limited the transcriptome analysis of the untreated control cells to those PSC-derived mdDAns in which PITX3 is induced independently of DKK3/WNT1 by effectively removing PITX3-/TH+ mdDAns from the analysis. However, because DKK3 induces PITX3 expression in a SNc subset of $\mathrm{TH}+$ mdDAns, it would have been essential to include both PITX3-/TH+ and PITX3 $+/ \mathrm{TH}+$ cells in the analysis to obtain insight into how DKK3/WNT1 induces PITX3 in TH+ SNc-mdDAns. Analysis of all $\mathrm{TH}+$ cells, independent of their PITX3 expression (for example by FACSpurification of mdDAns based on a TH reporter) would therefore have been more informative.

The authors' transcriptome analysis also demonstrated something else they did not discuss: in vitro samples primarily clustered according to the independent differentiation experiments, but not the treatment condition within those experiments, suggesting that experimental variance is a more important predictor of the differentiation outcome than the experimental treatment (Fukusumi et al., 2015, their Fig. 7D). Importantly, in one of the differentiation experiments (Con3/5_19 in their Fig. 7E), mdDAns were obtained that expressed mdDAn markers at levels more comparable to those of mdDAns in vivo, including high levels of rostrolateral mdDAn markers (Veenvliet and Smidt, 2014). These findings suggest that there are many variables besides the treatment condition (e.g., plating density, timing of treatments, passage number of starting culture) that influence the final resemblance of PSC-derived mdDAns to their in vivo counterpart, and it is important to analyze the effect of these conditions on the differentiation outcome.

Despite these caveats, the identification of a WNT modulator as a novel regulator of mdDAn development is of utmost interest, because fine-tuning of WNT cascades is critical for inducing the proper molecular signature of mdDAns and may influence mdDAn subtype (Are- nas, 2014). For example, excess WNT/ $\beta$ catenin signaling leads to improper progenitor specification, and SNc progenitors may be more susceptible to this (Nouri et al., 2015). In this light, DKK3 may act by fine-tuning the WNT signal and/or modulating the balance between canonical and noncanonical WNT signaling, thereby facilitating differentiation of progenitors toward SNc-mdDAns, while suppressing other fates. If this is the case, it follows that sequential application of WNT1 and DKK3 could be beneficial, first triggering WNT1-induced mdDA progenitor expansion followed by DKK3induced mdDAn differentiation. This should be tested in future studies, together with careful dosage titration.

Finally, the study is of critical importance to the field because of its experimental pipeline, which should be further expanded in future studies (Fig. 1). Due to the ever-increasing feasibility of identifying and characterizing small, defined subsets of cells in vivo, it is not a question of if, but rather how many subset-specific factors will be identified. Against this background, the authors' approach excellently demonstrates how initial clues from explorative neurodevelopmental studies should be used to develop differentiation protocols that efficiently and specifically generate SNc-mdDAns from PSCs.

In sum, the study by Fukusumi et al. (2015) emphasizes the necessity of studying mdDAn development in normal and mutant mice, and demonstrates how the systematic study of factors essential for in vivo SNc development can ultimately provide us with the proper recipe for safe stem cell therapy in PD.

\section{References}

Arenas E (2014) Wnt signaling in midbrain dopaminergic neuron development and regenerative medicine for Parkinson's disease. J Mol Cell Biol 6:42-53. CrossRef Medline

Bodea GO, Blaess S (2015) Establishing diversity in the dopaminergic system. FEBS Lett 89: 3773-3785. CrossRef Medline

Brichta L, Greengard P (2014) Molecular determinants of selective dopaminergic vulnerability in Parkinson's disease: an update. Front Neuroanat 8:152. CrossRef Medline

Brown A, Machan JT, Hayes L, Zervas M (2011) Molecular organization and timing of Wnt expression define cohorts of midbrain dopamine neuron progenitors in vivo. J Comp Neurol 519:2978-3000. CrossRef Medline

Fukusumi Y, Meier F, Götz S, Matheus F, Irmler M, Beckervordersandforth R, Faus-Kessler T, Minina E, Rauser B, Zhang J, Arenas E, Andersson E, Niehrs C, Beckers J, Simeone A, Wurst W, Prakash N (2015) Dickkopf 3 promotes the differentiation of a rostrolateral midbrain dopaminergic neuronal subset in vivo and from pluripotent stem cells in vitro in the mouse. J Neurosci 35:13385-13401. CrossRef Medline

Grealish S, Jönsson ME, Li M, Kirik D, Björklund A, Thompson LH (2010) The A9 dopamine neuron component in grafts of ventral mesencephalon is an important determinant for recovery of motor function in a rat model of Parkinson's disease. Brain 133:482-495. CrossRef Medline

Joksimovic M, Awatramani R (2014) Wnt/Bcatenin signaling in midbrain dopaminergic neuron specification and neurogenesis. J Mol Cell Biol 6:27-33. CrossRef Medline

Kriks S, Shim JW, Piao J, Ganat YM, Wakeman DR, Xie Z, Carrillo-Reid L, Auyeung G, Antonacci C, Buch A, Yang L, Beal MF, Surmeier DJ, Kordower JH, Tabar V, Studer L (2011) Dopamine neurons derived from human ES cells efficiently engraft in animal models of Parkinson's disease. Nature 480:547-551. CrossRef Medline

L'Honoré A, Coulon V, Marcil A, Lebel M, Lafrance-Vanasse J, Gage P, Camper S, Drouin J (2007) Sequential expression and redundancy of Pitx2 and Pitx3 genes during muscle development. Dev Biol 307:421-433. CrossRef Medline

Nouri N, Patel MJ, Joksimovic M, Poulin JF, Anderegg A, Taketo MM, Ma YC, Awatramani R (2015) Excessive WNT/beta-catenin signaling promotes midbrain floor plate neurogenesis, but results in vacillating dopamine progenitors. Mol Cell Neurosci 68:131-142. CrossRef Medline

Roessler R, Smallwood SA, Veenvliet JV, Pechlivanoglou P, Peng SP, Chakrabarty K, Groot-Koerkamp MJ, Pasterkamp RJ, Wesseling E, Kelsey G, Boddeke E, Smidt MP, Copray S (2014) Detailed analysis of the genetic and epigenetic signatures of iPSC-derived mesodiencephalic dopaminergic neurons. Stem Cell Reports 2:520-533. CrossRef Medline

Steinbeck JA, Studer L (2015) Moving stem cells to the clinic: potential and limitations for brain repair. Neuron 86:187-206. CrossRef Medline

Thompson L, Barraud P, Andersson E, Kirik D, Björklund A (2005) Identification of dopaminergic neurons of nigral and ventral tegmental area subtypes in grafts of fetal ventral mesencephalon based on cell morphology, protein expression, and efferent projections. J Neurosci 25:6467-6477. CrossRef Medline

Veeck J, Dahl E (2012) Targeting the WNT pathway in cancer: the emerging role of Dickkopf-3. Biochim Biophys Acta 1825: 18-28. CrossRef Medline

Veenvliet JV, Smidt MP (2014) Molecular mechanisms of dopaminergic subset specification: fundamental aspects and clinical perspectives. Cell Mol Life Sci 71:4703-4727. CrossRef Medline

Wurst W, Prakash N (2014) Wnt1-regulated genetic networks in midbrain dopaminergic neuron development. J Mol Cell Biol 6:34-41. CrossRef Medline 